\title{
Tumor suppressor REIC/Dkk-3 and its interacting protein SGTA inhibit glucocorticoid receptor to nuclear transport
}

\author{
TAKEHIRO IWATA $^{1}$, TAKUYA SADAHIRA ${ }^{1}$, KAZUHIKO OCHIAI $^{2}$, HIDEO UEKI $^{1}$, TAKANORI SASAKI ${ }^{3}$, \\ PENG HAUNG $^{1}$, MOTOO ARAKI ${ }^{1}$, TOYOHIKO WATANABE ${ }^{1}$, YASUTOMO NASU ${ }^{1}$ and MASAMI WATANABE ${ }^{1,4}$ \\ ${ }^{1}$ Department of Urology, Okayama University Graduate School of Medicine, Dentistry and Pharmaceutical Sciences, \\ Okayama 700-8558; ${ }^{2}$ School of Veterinary Nursing and Technology, Faculty of Veterinary Science, Nippon Veterinary and \\ Life Science University, Tokyo 180-8602; ${ }^{3}$ Collaborative Research Center for Okayama Medical Innovation Center (OMIC), \\ Okayama University Graduate School of Medicine, Dentistry and Pharmaceutical Sciences; ${ }^{4}$ Center for Innovative \\ Clinical Medicine, Okayama University Hospital, Okayama 700-8558, Japan
}

Received October 27, 2019; Accepted December 31, 2019

DOI: $10.3892 / \mathrm{etm} .2020 .8819$

\begin{abstract}
REIC/Dkk-3 is a tumor suppressor, and its expression is significantly downregulated in a variety of human cancer types. A previous study performed yeast two-hybrid screening and identified the small glutamine-rich tetratricopeptide repeat-containing protein $\alpha$ (SGTA), known as a negative modulator of cytoplasmic androgen receptor (AR) signaling, which is a novel interacting partner of REIC/Dkk-3. The previous study also indicated that the REIC/Dkk-3 protein interferes with the dimerization of SGTA and then upregulates the AR transport and signaling in human prostate cancer PC3 cells. Since the transport of some steroid receptors to nucleus is conducted similarly by dynein motor-dependent way, the current study aimed to investigate the role of SGTA and REIC/Dkk-3 in the transport of other glucocorticoid receptors (GR). In vitro reporter assays for the cytoplasmic GR transport were performed in human prostate cancer PC3 cells and 293T cells. As for the SGTA protein, a suppressive effect on the GR transport to the nucleus was observed in the cells. As for the REIC/Dkk-3 protein, an inhibitory effect was observed for the GR transport in PC3 cells. Under the depleted condition of SGTA by short-hairpin (sh)RNA, the downregulation of GR transport by REIC/Dkk-3 was significantly enhanced compared with the non-depleted condition in PC3 cells, suggesting a compensatory role of REIC/Dkk-3 in the SGTA mediated inhibition of GR transport. The current study therefore demonstrated that SGTA inhibited the cytoplasmic transport of GR in 293T and PC3 cells, and REIC/Dkk-3 also inhibited the cytoplasmic transport of GR in PC3 cells. These
\end{abstract}

Correspondence to: Professor Masami Watanabe, Center for Innovative Clinical Medicine, Okayama University Hospital, 2-5-1 Shikata-cho, Okayama 700-8558, Japan

E-mail: mwcorrespondence@gmail.com

Key words: REIC/Dkk-3, SGTA, glucocorticoid receptor, prostate cancer, androgen receptor results may be used to gain novel insight into the GR transport and signaling in normal and cancer cells.

\section{Introduction}

Prostate cancer development is characterized primarily by dependence on the androgen/androgen receptor (AR) axis (1). Therefore, therapeutics targeting this axis, such as androgen-deprivation therapy (ADT), are the standard treatments for advanced prostate cancer (2). However, the major concern with treatment of advanced prostate cancer is the development of resistance to ADT. ADT-resistant prostate cancer is still dependent on AR due to AR modifications, AR gene point mutations, upregulation of AR splice variants, and ligand-independent AR activation (3-5). Although the underlying androgen resistance mechanisms have been identified, these mechanisms only partially explain AR insensitivity.

Glucocorticoids are currently used to treat prostate cancer, as they suppress the secretion of adrenocorticotrophic hormone, resulting in reduced expression of adrenal androgen (6). Recently, elevated expression of the glucocorticoid receptor (GR) has been linked to prostate cancer resistance to ADT and disease progression $(7,8)$. In fact, a previous study observed GR upregulated expression in pathological tissue specimens from patients with androgen-independent prostate cancer (7). This is likely because AR can directly repress GR expression via a negative androgen response element in the GR promoter (9). GR can functionally replace AR by blocking AR signaling (9). These findings suggest that AR and GR potentially have significant overlap in their gene targets and interacting proteins $(7,10)$. Thus, novel and effective therapeutic agents are needed to treat prostate cancer by targeting not only AR but also GR.

The reduced expression in immortalized cells (REIC) gene, which was initially discovered as a tumor suppressor gene, is identical to Dickkopf-3 (Dkk-3), a member of the Dickkopf gene family (11). REIC/Dkk-3 is ubiquitously expressed in normal cells but significantly downregulated in various cancers, including prostate cancer (12). Many studies have shown that REIC/Dkk-3 downregulation is closely 
associated with the pathological malignancy of various cancer types $(12,13)$. An adenovirus vector carrying REIC/Dkk-3 selectively induced apoptosis in prostate cancer cells, but not in normal cells $(14,15)$. The induction of apoptosis is dependent on activation of c-jun- $\mathrm{NH}_{2}$ kinase and c-Jun by cellular endoplasmic reticulum stress signaling (16).

We previously demonstrated that small glutamine-rich tetratricopeptide repeat-containing protein $\alpha$ (SGTA) is a novel interaction partner of REIC/Dkk-3 (17). SGTA is ubiquitously expressed in almost all tissues, and recently characterized as a heat shock protein (Hsp)70- and Hsp90-associated co-chaperone that specifically downregulates AR signaling (18-21). Therefore, SGTA can influence the actions of androgens and be consequently involved in hormone-mediated cancer progression. AR signaling consists of AR maturation and transport to the nucleus via the dynein motor axis. SGTA dimerization stabilizes the AR complex and limits AR transport to the nucleus. REIC/Dkk-3 interferes with SGTA dimerization, promotes dynein-dependent AR transport to the nucleus, and subsequently upregulates AR signaling in human prostate cancer PC3 cells treated with dihydrotestosterone (17).

Although REIC/Dkk-3 may have a role in androgenindependent prostate cancer progression via regulation of $A R$ signaling, the ability of REIC/Dkk-3 to regulate other steroid hormone receptors remains unclear. We herein investigated the regulation of GR by REIC/Dkk-3 to further evaluate the mechanism by which REIC/Dkk-3 regulates steroid receptor transport in human prostate cancer cells.

\section{Materials and methods}

Cell culture and plasmid vectors. The human prostate cancer cell line PC3 and the human embryonic kidney cell line 293T were provided by the American Type Culture Collection. PC3 cells were maintained in Ham's F12 medium (Thermo Fisher Scientific, Inc.) supplemented with $10 \%$ fetal bovine serum, penicillin $(50 \mathrm{IU} / \mathrm{ml})$, and streptomycin $(50 \mu \mathrm{g} / \mathrm{ml})$ under a humidified atmosphere with $5 \% \mathrm{CO}_{2}$ at $37^{\circ} \mathrm{C}$. The $293 \mathrm{~T}$ cells were maintained in Dulbecco's modified Eagle's medium (DMEM; Thermo Fisher Scientific) for 18-24 h until the cells reached $60-80 \%$ confluence. The shRNA oligonucleotide (TG309472B; Origene) and matched control shRNA were transfected into PC3 and 293T cells. Cell lysates were obtained at the indicated time points for western blot analysis. As for construction of the plasmid vectors, the full length cDNA of human SGTA and human REIC/Dkk-3 were cloned into the pMACS Kk.HA-C vector (Miltenyi Biotec).

Western blot analysis. Both PC3 and 293T cells were transfected using Lipofectamine $3000^{\mathrm{TM}}$ transfection reagent (Thermo Fisher Scientific, Inc.) with $250 \mathrm{ng}$ of one of the following vectors: pMACS Kk.HA-C-SGTA, pMACS Kk.HA-C-REIC/Dkk-3, pGFP-V-RS containing SGTA-specific shRNA, or pMACS Kk.HA-C empty vector as the control. The cells were harvested at $48 \mathrm{~h}$ after transfection with the vectors. Approximately $5 \mu \mathrm{g}$ extracted proteins were subjected to Western blot analysis using the following specific primary antibodies: Monoclonal mouse anti-human REIC/Dkk-3 antibody (raised in our laboratory) (17), rabbit polyclonal anti-SGTA (cat. no. 11019-2-AP;
Protein Tech), and anti- $\beta$-actin (cat. no. 4967S; Cell Signaling Technology). The secondary antibodies used were horseradish peroxidase-conjugated anti-mouse IgG or anti-rabbit IgG (GE Healthcare). Western blot analysis was performed as described previously (13).

Analyses of GR translocation to the nucleus. Approximately $1.25 \times 10^{5}$ PC 3 cells and $5.0 \times 10^{5} 293 \mathrm{~T}$ cells on 24 -well plates were transfected with $100 \mathrm{ng}$ pMACS Kk.HA-C-SGTA, pMACS Kk.HA-C-REIC/Dkk-3, pGFP-V-RS containing SGTA-specific shRNA, pBIND-GR containing the GR ligand-biding domain and renilla luciferase reporter gene (cat. no. E158A; Promega), or pGL4.35(luc2P/9XGAL4 UAS/Hygro) containing the firefly luciferase reporter gene (cat. no. E137A; Promega). At $24 \mathrm{~h}$ post-transfection, the cells were treated for $24 \mathrm{~h}$ with $1 \mu \mathrm{M}$ dexamethasone (cat. no. D4902; Sigma) or the control (ethanol+DMEM) and then subjected to luciferase assays using the Dual-Luciferase ${ }^{\circledR}$ Reporter Assay System (Promega) (22). Luciferase was added to the culture media for $15 \mathrm{~min}$, after which firefly luminescence was measured using the Multi-detection Reader Flex Station 3 (Molecular Device). Subsequently, Stop and Glo Reagent was added to the culture medium, and renilla luminescence was measured immediately. Relative promoter activity was calculated as the ratio of firefly luciferase to renilla luciferase luminescence. All transfection mixes were balanced with the appropriate empty vectors in terms of the ratio of the expression vectors and total plasmids. The firefly/renilla dual luciferase vector encoding the Gal4 DNA-binding domain fused to the glucocorticoid receptor ligand-binding domain allows measurement of both firefly and renilla luciferase activity in the same sample with high sensitivity and linearity. The dual luciferase reporter assay is a screening tool to detect the simultaneous expression and measurement of each reporter enzyme within a single system. Normalizing the activity of the experimental reporter to the activity of the internal control minimizes experimental variability caused by differences in cell viability or transfection efficiency. Furthermore, other variabilities such as differences in pipetting volumes, cell lysis efficiency and assay efficiency can be effectively eliminated. Thus, dual-reporter assays often allow more reliable interpretation of the experimental data by reducing extraneous influences (23).

Statistical analyses. The data are presented as means \pm standard error. The unpaired Student's t-test was performed for comparisons between two groups. $\mathrm{P}<0.05$ was considered to indicate a statistically significant difference. Statistical analyses were performed using StatView version 4.5 software (Abacus Concept).

\section{Results}

Expression of SGTA and REIC/Dkk-3 in prostate cancer PC3 and 2937 cell lines. To assess the expression of SGTA and REIC/Dkk-3 proteins, we performed Western blot analyses in PC3 and 293T cells. SGTA and REIC/Dkk-3 proteins were overexpressed in PC3 and 293T cells by transfection of the vectors containing the human SGTA and human REIC genes, respectively (Fig. 1). SGTA expression was suppressed in 

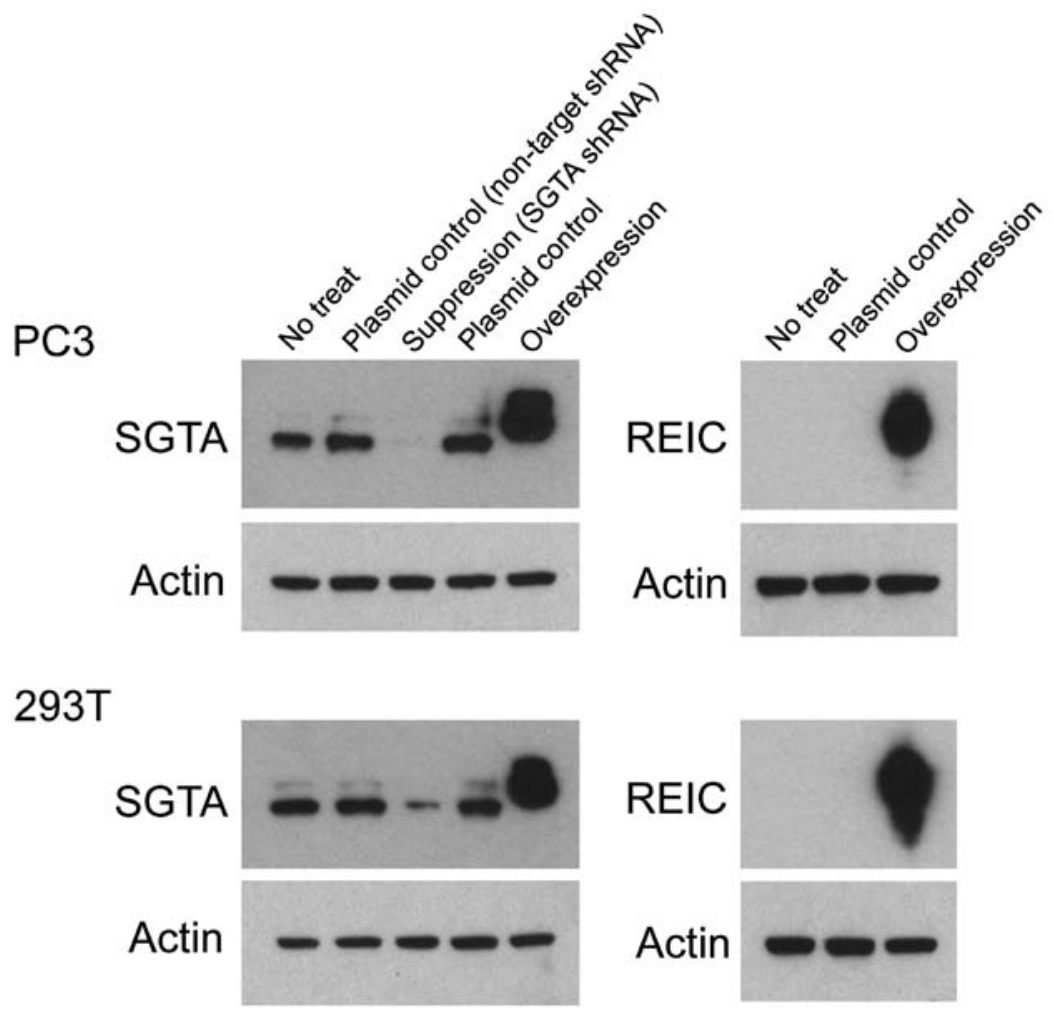

Figure 1. SGTA and REIC/Dkk-3 were overexpressed by transfection of the plasmid encoding SGTA and REIC genes in PC3 and 293T cells, and verified using western blot analysis. SGTA expression was suppressed by transfection of SGTA-specific shRNA in PC3 and 293T cells, and also verified. Actin expression was shown as a loading control. SGTA, small glutamine-rich tetratricopeptide repeat-containing protein $\alpha$; sh, short-hairpin.

PC3 and 293T cells by transfection of the pGFP-V-RS vector containing SGTA-specific shRNA (Fig. 1).

Inhibitory effects of SGTA and REIC/Dkk-3 in GR signaling. To investigate the roles of SGTA and REIC/Dkk-3 in GR transport to nucleus, we performed in vitro luciferase reporter assays for the cytoplasmic GR transport in human prostate cancer PC3 cells and 293T cells. As for the SGTA protein, the amount of GR transport to nucleus was oppositely affected in comparison to the levels of SGTA expression in the both cells (Figs. 2A and 3A). As for the REIC/Dkk-3 protein, the GR transport was inhibited by REIC/Dkk-3 overexpression only in the PC3 cells (Figs. 2B and 3B). These results indicate that both of the SGTA and REIC/Dkk-3 inhibit the cytoplasmic transport of GR to nucleus in human prostate cancer PC 3 cells.

Inhibitory effect of REIC/Dkk-3 on the GR transport is augmented under the SGTA depleted condition. We previously disclosed that intracellular REIC/Dkk-3 interacts with SGTA and the interaction modify the cytoplasmic androgen receptor (AR) transport in the PC3 cells treated with dihydrotestosterone (17). Since we herein demonstrated that both SGTA and REIC/Dkk-3 inhibit the GR transport to nucleus in human prostate cancer PC3 cells, it is conceivable that the expressional state of REIC/Dkk-3 and SGTA protein may modify their inhibitory effects on GR transport to nucleus in the cells. To examine the mutual effects of SGTA and REIC/Dkk-3 on each other in terms of GR transport, we simultaneously manipulated the expression levels of these proteins in PC3 and 293T cells. Under the depleted condition of SGTA by shRNA, the downregulation of GR transport by REIC/Dkk-3 was significantly augmented in comparison to the non-depleted condition, indicating a compensatory association of REIC/Dkk-3 in the SGTA mediated inhibition of GR transport (Figs. 2C and 3C). On the other hands, under the overexpressed condition of SGTA, the inhibitory effect of REIC/Dkk-3 on the GR transport was disappeared in PC3 cells (Fig. 2C).

\section{Discussion}

The present study demonstrated that REIC/Dkk-3 expression significantly inhibited GR transport to the nucleus in human prostate cancer PC3 cells. After shRNA-mediated depletion of SGTA expression, downregulation of GR transport by REIC/Dkk-3 was significantly enhanced compared to the control cells with normal SGTA expression, suggesting a compensatory role of REIC/Dkk-3 for the inhibitory function of SGTA in GR transport. We are the first to demonstrate that both REIC/Dkk-3 and SGTA inhibit cytoplasmic transport of GR to the nucleus in human prostate cancer cells.

Steroid hormone receptors are ligand-dependent transcription factors that require dynamic, ordered assembly of the chaperone and co-chaperone machinery to obtain a functional conformation (19). Hsp70 and Hsp90 are key factors in this process and several Hsp70- and Hsp90-associated co-chaperones interact with receptor-chaperone complexes to functionally affect a wide variety of steps within the receptor-folding process $(24,25)$. Referring the published reports, we herein proposed the role of SGTA and 
A

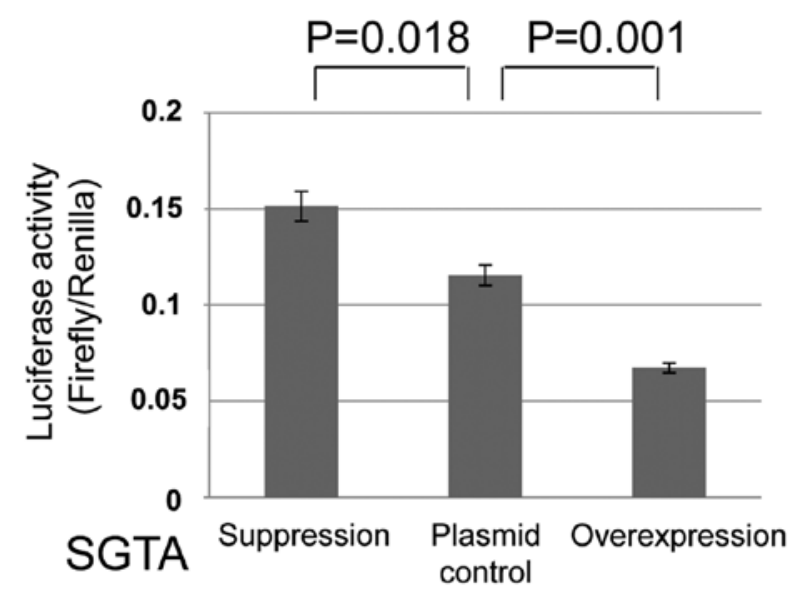

B

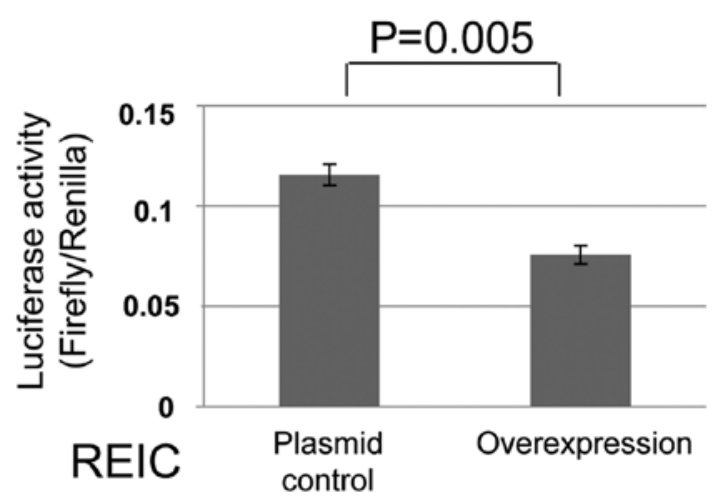

C
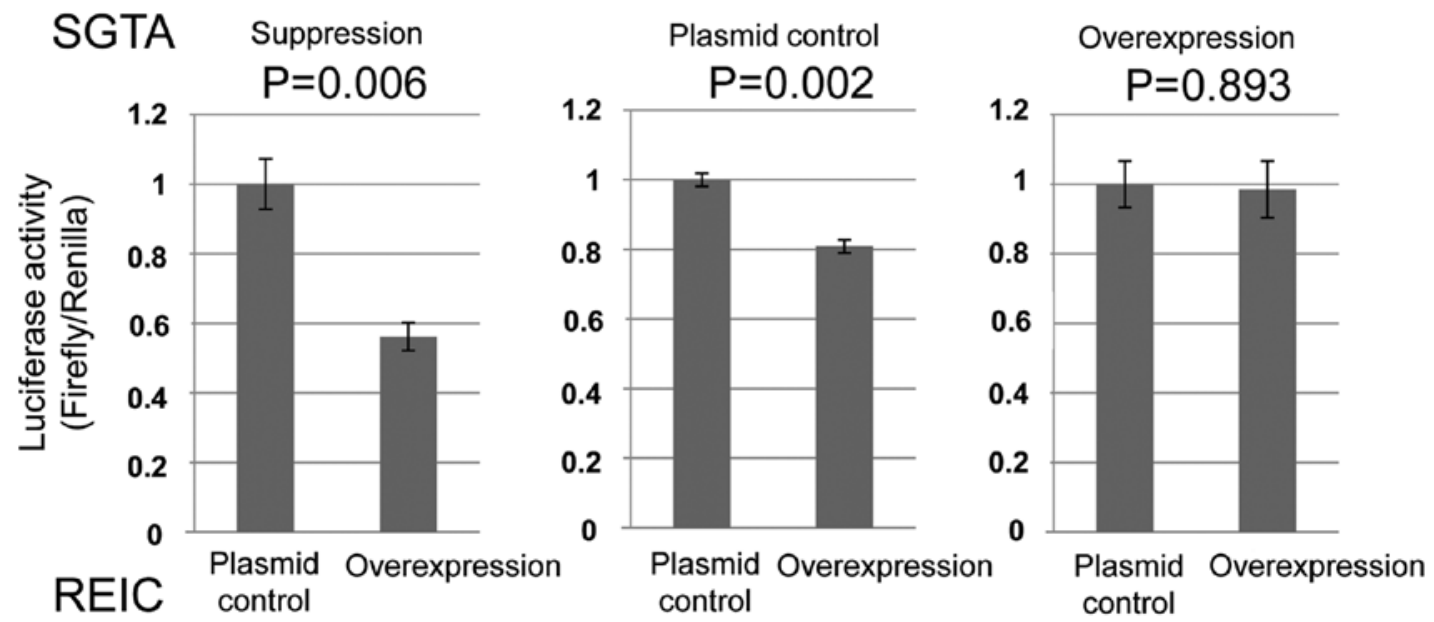

Figure 2. The effects of the SGTA and REIC/Dkk-3 protein on cytoplasmic GR transport to nucleus in PC3 cells. (A) The effects based on the SGTA expression levels on GR transport. (B) The effects of REIC/Dkk-3 overexpression on GR transport. (C) The modified effects of the REIC/Dkk-3 overexpression on GR transport according to SGTA expression levels. The renilla luciferase expression pBIND vector was used to normalize the transfection control for the firefly luciferase assay. The luciferase activity in the cells was measured at $48 \mathrm{~h}$ after transfection and calculated as the ratio of firefly to renilla luciferase luminescence. SGTA, small glutamine-rich tetratricopeptide repeat-containing protein $\alpha$; GR, glucocorticoid receptors.

REIC/Dkk-3 in cytoplasmic GR transport to nucleus (Fig. 4) (17,20,21,26-29). GR transport consists of the GR maturation and GR complex transport to nucleus via the dynein motor axis. In this study, we demonstrated that both SGTA and REIC/Dkk-3 inhibit the GR transport to nucleus in human prostate cancer PC 3 cells. Although the PC 3 cell line was reported to be AR-negative, Akimirah et al demonstrated that treatment of the PC 3 cell line with dihydrotestosterone resulted in measurable increases in the AR protein levels and considerable nuclear accumulation (30). We previously indicated that cytoplasmic REIC/Dkk-3 upregulates dynein motor-dependent AR transport via its interaction with the co-chaperone SGTA. In addition, the interaction between REIC/Dkk-3 and SGTA modify the degree of cytoplasmic AR transport in the PC3 cells treated with dihydrotestosterone (17). Based on these findings, it is likely that the 
A

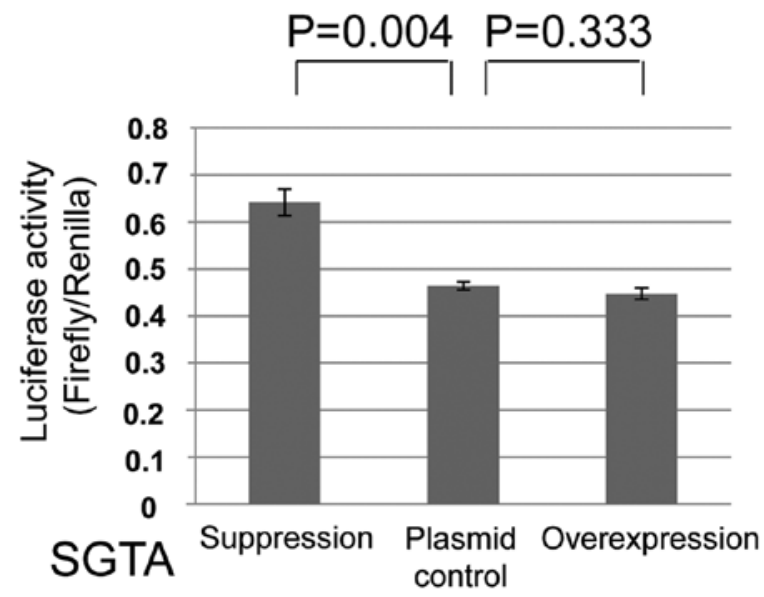

B

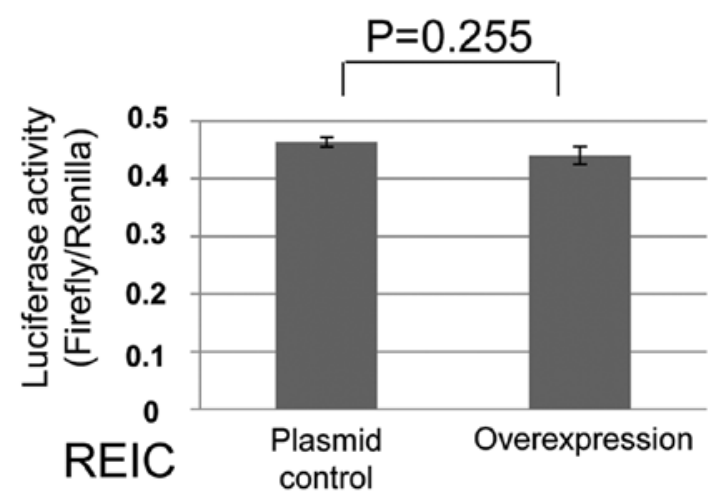

C
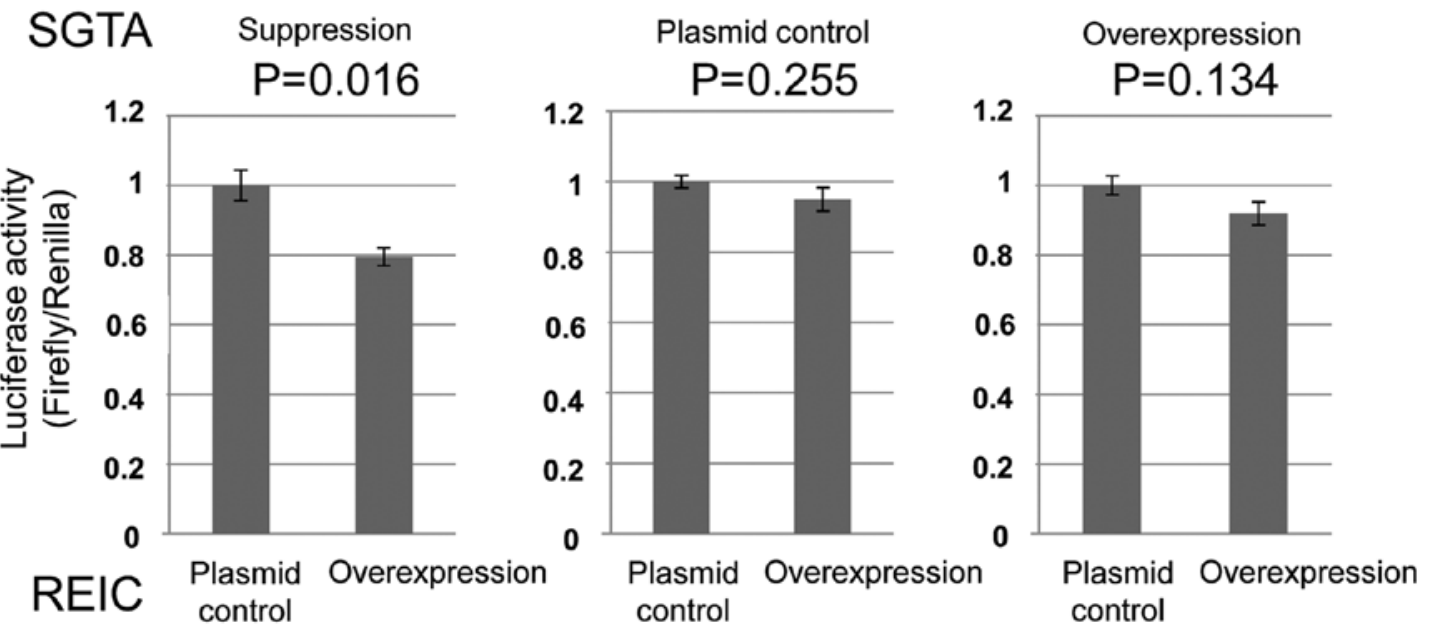

Figure 3. The effects of the SGTA and REIC/Dkk-3 protein on cytoplasmic GR transport to nucleus in 293T cells. (A) The effects based on the SGTA expression levels on GR transport. (B) The effects of the REIC/Dkk-3 overexpression on GR transport. (C) The modified effects of the REIC/Dkk-3 overexpression on GR transport according to the SGTA expression levels. The renilla luciferase expression pBIND vector was used to standardize the transfection efficiency. The luciferase activity in the cells was measured at $48 \mathrm{~h}$ after transfection and calculated as the ratio of firefly to renilla luciferase luminescence. SGTA, small glutamine-rich tetratricopeptide repeat-containing protein $\alpha$; GR, glucocorticoid receptors.

interaction between REIC/Dkk-3 and SGTA protein modifies their own inhibitory roles of GR transport in the cells.

The precise molecular mechanism by which REIC/Dkk-3 suppresses GR transport remains to be determined. REIC/Dkk-3 and SGTA were shown cytoplasmic localization with a similar distribution pattern. We previously reported that $\mathrm{AR}$ was predominantly located in the cytoplasm when SGTA was expressed and the expression of REIC/DKK-3 facilitated AR transport to nucleus (17). Furthermore, REIC/Dkk-3 binds to SGTA, and their interaction involves the $78 \mathrm{~N}$-terminal amino acids of both proteins (17). Based on the binding, REIC/Dkk-3 interferes with the dimerization of SGTA and abolishes SGTA dependent negative regulation of AR transport, resulting in enhanced AR signaling in the $\mathrm{PC} 3$ prostate cancer cells. However, in the present study, REIC/Dkk-3 unexpectedly inhibited the GR transport to the nucleus and enhanced the degree of inhibition under the 


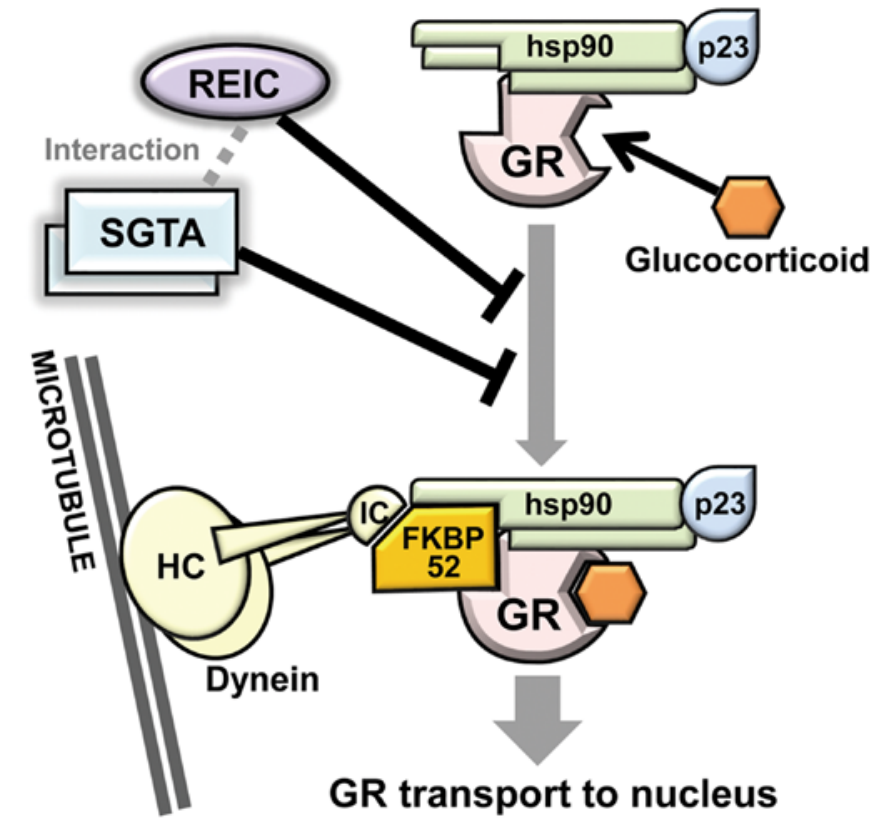

Figure 4. The proposed role of SGTA and REIC/Dkk-3 in cytoplasmic GR transport to nucleus is shown. GR transport consists of the GR maturation and the GR complex transport to nucleus via the dynein motor axis. The results of the current study demonstrated that SGTA and REIC/Dkk-3 inhibit the GR transport to nucleus in human prostate cancer PC3 cells. It has been previously indicated that REIC/Dkk-3 interacts with SGTA and the interaction modify the cytoplasmic androgen receptor transport in the PC3 cells. The interaction between REIC/Dkk-3 and SGTA protein may modify their own inhibitory roles of GR transport in the cells. SGTA, small glutamine-rich tetratricopeptide repeat-containing protein $\alpha$; GR, glucocorticoid receptors.

SGTA depleted condition. As for the GR transport, it is likely that REIC/Dkk-3 protein possesses similar molecular activity with SGTA and the proteins work as co-effectors in the process of GR transport inhibition. Further experiments are necessary to reveal whether these two proteins interact with each other to cooperate in the inhibition of GR transport, and whether the proteins cooperate in regulating their own functions.

A recent study suggested that GR expression is associated with resistance to the ADT in androgen-independent prostate cancer $(7,8)$. GR is elevated in bone metastases of prostate cancer patients following enzalutamide therapy, and high GR expression is associated with poor prognosis (7). In addition, GR is upregulated in LNCaP/AR xenografts and in the cell lines that are able to grow in the presence of enzalutamide, suggesting that GR can drive the expression of AR target genes (7). These findings indicate a significant role of GR signaling in the resistance to anti-androgen therapy. In this study, we demonstrated that intracellular REIC/Dkk-3 is potentially an inhibitor of GR transport. In our previous study, we showed that REIC/Dkk-3 activates AR transport and signaling by binding to and interfering with SGTA, a negative regulator of cytoplasmic AR transport (17). Therefore, intracellular REIC/Dkk-3 has two aspects of a GR inhibitor and AR activator as a modifier of the steroid signaling.

The present study has some limitations. We only used the PC 3 cell line which was reported androgen-independent prostate cancer cells, and further study is needed to verify the effect of SGTA and REIC/Dkk-3 proteins on the GR axis in the other cell lines (e.g. LNCaP or DU145). We did not investigate the changes of $\mathrm{AR}$ and GR cellular distributions depending on expression level of SGTA and REIC/Dkk-3, and also not investigate the protein-protein interactions involved in this process. Despite these limitations, this study could be significant for developing novel therapeutic approach for androgen-independent prostate cancer.

In conclusion, REIC/Dkk-3 and SGTA inhibit cytoplasmic transport of GR to the nucleus in human prostate cancer PC3 cells. The findings could be of significance for understanding GR signaling in the androgen-independent prostate cancer cells. The mechanism of interaction between REIC/Dkk-3 and SGTA associated with the GR complex provides new insights for developing novel medicine targeting GR signaling.

\section{Acknowledgements}

The authors thank Ms. Fukasa Oonari (Center for Innovative Clinical Medicine, Okayama University Hospital, Okayama 700-8558, Japan) and Ms. Shun-Ai Li (Department of Neuroscience, Okayama University Graduate School of Medicine, Dentistry and Pharmaceutical Sciences, Okayama 700-8558, Japan) for their valuable assistance with the acquisition and interpretation of the data in the present study.

\section{Funding}

The current study was supported by scientific research grants from the Ministry of Education, Culture, Sports, Science and Technology of Japan, and by scientific research grants from the Teraoka Scholarship Foundation (JSPS KAKENHI grant nos. JP19H01064 and JP18K09167).

\section{Availability of data and materials}

The datasets used and/or analyzed during the current study are available from the corresponding author on reasonable request.

\section{Authors' contributions}

TI wrote the manuscript and performed the experiments. TSad made substantial contributions to the conception and design of the present study and the interpretation of data. HU made substantial contributions to the acquisition of the data and interpretation of data in the present study. PH, MA and TW made substantial contributions to the interpretation of data in the present study. KO, TSas, MW and YN made substantial contributions to the conception and design of the study, critical point of discussion and the completion of the manuscript. All authors read and approved the final manuscript.

\section{Ethics approval and consent to participate}

Not applicable.

\section{Patients consent for publication}

Not applicable.

\section{Competing interests}

The authors declare that they have no competing interests. 


\section{References}

1. Shiota M, Fujimoto N, Kashiwagi E and Eto M: The role of nuclear receptors in prostate cancer. Cells 8: 602, 2019.

2. Shiota M and Eto M: Current status of primary pharmacotherapy and future perspectives toward upfront therapy for metastatic hormone-sensitive prostate cancer. Int J Urol 23: 360-369, 2016

3. Kim JY, Yu J, Abdulkadir SA and Chakravarti D: KAT8 regulates androgen signaling in prostate cancer cells. Mol Endocrinol 30 925-936, 2016

4. Thadani-Mulero M, Portella L, Sun S, Sung M, Matov A, Vessella RL, Corey E, Nanus DM, Plymate SR and Giannakakou P: Androgen receptor splice variants determine taxane sensitivity in prostate cancer. Cancer Res 74: 2270-2282, 2014.

5. Korpal M, Korn JM, Gao X, Rakiec DP, Ruddy DA, Doshi S, Yuan J, Kovats SG, Kim S, Cooke VG, et al: An F876L mutation in androgen receptor confers genetic and phenotypic resistance to MDV3100 (enzalutamide). Cancer Discov 3: 1030-1043, 2013

6. Ndibe C, Wang CG and Sonpavde G: Corticosteroids in the management of prostate cancer: A critical review. Curr Treat Options Oncol 16: 6, 2015.

7. Arora VK, Schenkein E, Murali R, Subudhi SK, Wongvipat J, Balbas MD, Shah N, Cai L, Efstathiou E, Logothetis C, et al Glucocorticoid receptor confers resistance to Antiandrogens by bypassing androgen receptor blockade. Cell 155: 1309-1322, 2013.

8. Isikbay M, Otto K, Kregel S, Kach J, Cai Y, Vander Griend DJ, Conzen SD and Szmulewiz RZ: Glucocorticoid receptor activity contributes to resistance to androgen-targeted therapy in prostate cancer. Horm Cancer 5: 72-89, 2014

9. Xie N, Cheng H, Lin D, Liu L, Yang O, Jia L, Fazli L, Gleave ME Wang Y, Rennie P and Dong X: The expression of glucocorticoid receptor is negatively regulated by active androgen receptor signaling in prostate tumors. Int J Cancer 136: E27-E38, 2015.

10. Lempiäinen JK, Niskanen EA, Vuoti KM, Lampinen RE, Göös H, Varjosalo M and Palvimo JJ: Agonist-specific protein interactomes of glucocorticoid and androgen receptor as revealed by proximity mapping. Mol Cell Proteomics 16 $1462-1474,2017$

11. Tsuji T, Miyazaki M, Sakaguchi M, Inoue $\mathrm{Y}$ and Namba $\mathrm{M}$ : A REIC gene shows down-regulation in human immortalized cells and human tumor-derived cell lines. Biochem Biopsy Res Commum 268: 20-24, 2000.

12. Hirata T, Watanabe M, Kaku H, Kobayashi Y, Yamada H, Sakaguchi M, Takei K, Huh NH, Nasu Y and Kumon H. REIC/Dkk-3-encoding adenoviral vector as a potentially effective therapeutic agent for bladder cancer. Int J Oncol 41: 559-564, 2012.

13. Watanabe M, Sakaguchi M, Kinoshita R, Kaku H, Ariyoshi Y, Ueki H, Tanimoto R, Ebara S, Ochiai K, Futami J, et al: A novel gene expression system strongly enhances the anticancer effects of a REIC/Dkk-3-encoding adenoviral vector. Oncol Rep 31: 1089-1095, 2014

14. Abarzua F, Sakaguchi M, Tanimoto R, Sonegawa H, Li DW, Edamura K, Kobayashi T, Watanabe M, Kashiwakura T, Kaku H, et al: Heat shock proteins play a crucial role in tumor-specific apoptosis by REIC/Dkk-3. Int J Mol Med 20: 37-43, 2007.
15. Kumon H, Sasaki K, Ariyoshi Y, Sadahira T, Ebara S, Hiraki T, Kanazawa S, Yanai H, Watanabe M and Nasu Y: Ad-REIC gene therapy: Promising results in a patient with metastatic CRPC following chemotherapy. Clin Med Insights Oncol 9: 31-38, 2015.

16. Abarzua F, Sakaguchi M, Takaishi M, Nasu Y, Kurose K, Ebara S, Miyazaki M, Namba M, Kumon $\mathrm{H}$ and Huh NH: Adenovirus-mediated overexpression of REIC/Dkk-3 selectively induces apoptosis in human prostate cancer cells through activation of c-Jun-NH2-kinase. Cancer Res 65: 9617-9622, 2005.

17. Ochiai K, Morimatsu M, Kato Y, Ishiguro-Oonuma T, Udagawa C, Rungsuriyawiboon O, Azakami D, Michishita M, Ariyoshi Y, Ueki H, et al: Tumor suppressor REIC/Dkk-3 and co-chaperone SGTA: Their interaction and roles in the androgen sensitivity. Oncotaget 7: 3283-3296, 2016.

18. Liu FH, Wu SJ, Hu SM, Hsiao CD and Wang C: Specific interaction of the $70-\mathrm{kDa}$ heat shock cognate protein with the tetratricopeptide repeats. J Bio Chem 247: 34425-34432, 1999.

19. Paul A, Garcia YA, Zierer B, Patwardhan C, Gutierrez O, Hildenbrand Z, Harris DC, Balsiger HA, Sivils JC, Johnson JL, et al: The cochaperone SGTA demonstrates regulatory specificity for the androgen, glucocorticoid, and progesterone receptors. J Biol Chem 289: 15297-15308, 2014.

20. Buchanan G, Ricciardeli C, Harris JM, Prescott J, Yu ZC, Jia L, Butler LM, Marshall VR, Scher HI, Gerald WL, et al: Control of androgen receptor signaling in prostate cancer by the cochaperone small glutamine rich tetraticopeptide repeat containing protein alpha. Cancer Res 67: 10087-10096, 2007.

21. Philp LK, Butler MS, Hichey TE, Bulter LM, Tilley WD and Day TK: SGTA: A new player in the molecular co-chaperone game. Horm cancer 4: 343-357, 2013.

22. Kaczmarczyk SJ and Green JE: A single vector containing modified cre recombinase and LOX recombination sequences for inducible tissue-specific amplification of gene expression. Nucleic Acids Res 29: E56, 2001.

23. Dual-Luciferase ${ }^{\circledR}$ Reporter Assay System Technical Manual. https://www.promega.com/-/media/files/resources/ protocols/technical-manuals/0/dual-luciferase-reporter-assay-systemprotocol.pdf

24. Echeverria PC and Picard D: Molecular chaperones, essential partners of steroid hormone receptors for activity and mobility. Biochim Biophys Acta 1803: 641-649, 2010.

25. Smith DF and Toft DO: Minireview: The intersection of steroid receptors with molecular chaperones: Observations and questions. Mol Endocrinol 22: 2229-2240, 2008.

26. Pratt WB, Galigniana MD, Morishima Y and Murphy PJ: Role of molecular chaperones in steroid receptor action. Essays Biochem 40: 41-58, 2004

27. Grad I and Picard D: The glucocorticoid responses are shaped by molecular chaperones. Mol Cell Endocrinol 275: 2-12, 2007.

28. Pérez MH, Cormack J, Mallinson D and Mutungi G: A membrane glucocorticoid receptor mediates the rapid/non-genomic actions of glucocorticoids in mammalian skeletal muscle fibres. J Physiol 591: 5171-5185, 2013.

29. Cato L, Neeb A, Brown M and Cato AC: Control of steroid receptor dynamics and function by genomic actions of the cochaperones p23 and Bag-1L. Nucl Recept Signal 12: e005, 2014.

30. Akimirah F, Chen J, Basrawala Z, Xin H and Choubey D: DU-145 and PC-3 human prostate cancer cell lines express androgen receptor: Implications for the androgen receptor functions and regulation. FEBS Lett 2294-2300, 2006. 